\section{(6) OPEN ACCESS}

REVIEW

\title{
The differential diagnosis of Huntington's disease- like syndromes: 'red flags' for the clinician
}

\author{
Davide Martino, ${ }^{1,2}$ Maria Stamelou, ${ }^{3}$ Kailash P Bhatia ${ }^{3}$
}

\begin{abstract}
- Additional materials are published online only. To view these files please visit the journal online (http://dx.doi. org/10.1136/jnnp-2012302532).

${ }^{1}$ Neuroscience \& Trauma Centre, Barts and The London School of Medicine and Dentistry, Queen Mary University London, London, UK ${ }^{2}$ Queen Elizabeth Hospital, South London NHS Trust, Woolwich, London, UK ${ }^{3}$ Sobell Department of Motor Neuroscience and Movement Disorders, UCL Institute of Neurology, Queen Square, London, UK
\end{abstract}

Correspondence to Dr Davide Martino, Blizard Institute, Neuroscience \& Trauma Centre, Queen Mary University of London, 4 Newark Street, London E1 2AT, UK; d.martino@qmul.ac.uk

Received 3 June 2012 Revised 17 July 2012 Accepted 1 August 2012 Published Online First 19 September 2012

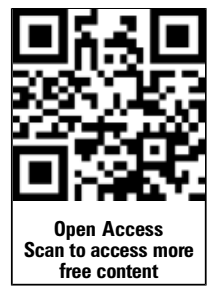

To cite: Martino $D$, Stamelou M, Bhatia KP. I Neurol Neurosurg Psychiatry 2013:84: 650-656.

\section{ABSTRACT}

A growing number of progressive heredodegenerative conditions mimic the presentation of Huntington's disease (HD). Differentiating among these HD-like syndromes is necessary when a patient with a combination of movement disorders, cognitive decline, behavioural abnormalities and progressive disease course proves negative to the genetic testing for HD causative mutations, that is, IT15 gene trinucleotide-repeat expansion. The differential diagnosis of HD-like syndromes is complex and may lead to unnecessary and costly investigations. We propose here a guide to this differential diagnosis focusing on a limited number of clinical features ('red flags') that can be identified through accurate clinical examination, collection of historical data and a few routine ancillary investigations. These features include the ethnic background of the patient, the involvement of the facio-bucco-lingual and cervical district by the movement disorder, the cooccurrence of cerebellar features and seizures, the presence of peculiar gait patterns and eye movement abnormalities, and an atypical progression of illness. Additional help may derive from the cognitivebehavioural presentation of the patient, as well as by a restricted number of ancillary investigations, mainly MRI and routine blood tests. These red flags should be constantly updated as the phenotypic characterisation and identification of more reliable diagnostic markers for HD-like syndromes progress over the following years.

\section{INTRODUCTION}

Huntington's disease (HD) is the most common hereditary neurodegenerative illness with onset before mid-life and a distinct phenotype characterised by movement disorders (mainly chorea, dystonia and Parkinsonism), incoordination, cognitive deterioration and behavioural changes. Progressive neural cell loss within cortico-striato-thalamocortical circuits, predominantly in the neostriatum (caudate and putamen), leads to this clinical presentation. ${ }^{1}$

HD is a trinucleotide-repeat disorder with autosomal dominant mode of inheritance. The causative mutation is a prolongation (above 35 repeats) of a trinucleotide (CAG) stretch within the IT15 gene, associated with full penetrance when this exceeds 40 and incomplete penetrance between 36 and 39. As in several other trinucleotide-repeat disorders, there is a strong association between repeat length and age at disease onset, with higher repeat length leading to earlier onset. Paternal transmission facilitates this anticipation phenomenon. Age at onset is strongly linked to the clinical expression of both motor and cognitive features of the disease. ${ }^{1}$ Juvenile HD (ie, with onset before age 20) is dominated by a hypokinetic-rigid syndrome (Westphal variant) and a more rapid course, whereas adult-onset HD classically presents with a choreatic syndrome and a less severe progression of motor and non-motor features. ${ }^{1}$

An increasing number of progressive heredodegenerative illnesses may mimic the presentation of HD. It has been estimated that between $1 \%$ and $7 \%$ of subjects with a HD-like syndrome do not have HD. ${ }^{2}$ Given the relationship between motor phenotype and age at onset in HD, these HD-like syndromes are, with some exceptions, either diseases manifesting with a predominantly choreatic syndrome and onset in adulthood (online supplementary table 1a) or diseases presenting with predominant dystonia and Parkinsonism and onset in the first 2 decades (online supplementary table $1 b$ ). The comprehensive understanding of the phenomenology and diagnosis of progressive HD-like syndromes is crucial for an efficient and cost-effective diagnostic work-up and guides further genetic testing once the diagnosis of HD has been genetically excluded. This work-up may include thorough clinical examination, neuropsychological and neuropsychiatric assessment, metabolic screening, imaging and electrophysiology.

To date, progressive hereditary HD-like syndromes were classified mainly according to their mode of inheritance. Although this criterion can be very helpful in the presence of a well-established family history, it may not be practically useful in the numerous cases in which family history is unavailable or inaccurate. In this review, we discuss the differential diagnosis of $\mathrm{HD}$ focusing on a restricted number of clinical features ('red flags') that might help clinicians to narrow the spectrum of working diagnoses and improve efficiency and cost-effectiveness of the diagnostic process.

\section{ETHNICITY}

HD and the majority of progressive HD-like syndromes can be encountered in most geographical areas and ethnic groups. There are, however, a few important exceptions to consider in the differential diagnosis.

Huntington's disease-like 2 (HDL2) is an autosomal dominant disorder described exclusively in families of Southern African origins, with the exception of one Brazilian family of Spanish/ Portuguese ancestry. This condition accounts for about $0.7 \%$ of all HD-like presentations. HDL2 is caused by a CTG stretch in the JPH3 gene coding 
for junctophilin-3, leading to ubiquitin-immunoreactive intranuclear inclusions in the cortex and neuronal loss in the striatum and cortex. Unlike in HD, repeat expansions are more unstable and the anticipation phenomenon is more likely when the disorder is maternally inherited. HDL2 starts at the same age as HD, but can present either in a Parkinsonian or in a choreatic form. ${ }^{3}$ The choreatic form is strikingly similar to typical adult-onset HD, although both can present with cognitive deterioration, psychiatric features, eye movement abnormalities and MRI changes highly similar to HD. Overall, HDL2 should be suspected in every patient of South African ancestry with a HD-like presentation, accounting for $24 \%-50 \%$ of these cases. ${ }^{3}$

A HD-like syndrome in patients of Japanese ethnicity should raise the suspicion of dentato-rubro-pallido-luysian atrophy (DRPLA), another autosomal dominant disorder (summarised in detail below). In Japan, the prevalence of DRPLA is estimated to be similar to the prevalence of HD. DRPLA has been reported in non-Japanese patients, and a single haplotype has been found in both Japanese and Caucasian patients, in line with a common founder effect shared across ethnic groups. ${ }^{4}$

Other conditions entering the differential diagnosis with HD could be more represented in some geographical areas than in others. Neuroferritinopathy is an autosomal dominant disorder presenting with progressive adult-onset chorea or dystonia initially affecting the limbs and mild cognitive deterioration. Molecular genetic testing for the FTL gene, coding for the ferritin light chain, confirms this diagnosis. ${ }^{5}$ This disorder was initially described in Cumbria, a region in the northwest of England. ${ }^{5}$ The majority of reported cases were originally from this region. Additional families reported in France and an additional North-American patient with German ancestry do not exclude the possibility of a founder effect in northwestern Europe. ${ }^{6}$ Other examples of ethnic segregation in HD-like syndromes include spino-cerebellar ataxia 8 , especially common in Finland, ${ }^{7}$ and ataxia with oculomotor apraxia (AOA) type 2, mainly seen in French-Canadian and Anglo-Norman populations. $^{8}$

\section{BODY SITE DISTRIBUTION OF MOTOR SYMPTOMS}

Most progressive HD-like syndromes display a generalised movement disorder, involving in different combinations face, neck, torso and limbs. A disabling involvement of the facio-bucco-linguo-masticatory region should, however, lead the clinician towards a few specific conditions.

HD-like syndromes presenting in adulthood with chorea or other movement disorders, including dystonia and tics, with marked involvement of the facio-bucco-linguo-masticatory region comprise 'neuroacanthocytosis syndromes', mainly chorea-acanthocytosis and McLeod syndrome. Chorea-acanthocytosis is an autosomal recessive disorder caused by mutations in the VPS13A gene coding for chorein, a large protein implicated in intracellular protein sorting. Facio-bucco-linguo-masticatory chorea, dystonia and dyskinesias are typically precipitated by feeding, with tongue protrusion and self-mutilating tongue and lip biting. ${ }^{9}$ These patients may also present with violent neck spasms with sudden flexion/extension that can lead to characteristic head drop and head banging (see online supplementary video 1). ${ }^{10}$ Vocalisations and dysarthria are also present, whereas Parkinsonism is rare. ${ }^{10}$ The co-occurrence of areflexia, weakness and muscle wasting, suggestive of concomitant peripheral neuropathy and/or myopathy, may further help in the diagnosis. ${ }^{11}$

McLeod syndrome is named after a Harvard medical student, Hugh McLeod, who did not express the Kell antigen on erythrocytes, as all affected male subjects with this condition. It is an $\mathrm{X}$ linked recessive disorder, present in most geographical regions, caused by different mutations in the XK gene. ${ }^{11}$ McLeod syndrome is a multi-system disorder, affecting basal ganglia, muscles, peripheral nerves and myocardium. ${ }^{11}$ It presents with chorea that may involve the facio-buccal region and vocalisations, but almost never with tongue or lip biting, dysphagia, or Parkinsonism; ${ }^{11}$ feeding dystonia has also been reported. ${ }^{12}$ Importantly, $60 \%$ of patients develop a cardiomyopathy with atrial fibrillation and risk of cardiac sudden death, thus requiring periodical cardiac monitoring.

When the movement disorder is mainly characterised by early dystonia or a hypokinetic-rigid syndrome with relevant facio-bucco-lingual involvement, the main differential diagnosis to consider is pantothenate kinase-associated neurodegeneration (PKAN), an autosomal recessive disorder with a frequency of 13 per million, caused by mutations of the PANK2 gene coding for pantothenate kinase, an enzyme relevant to the synthesis of coenzyme A from vitamin $\mathrm{B}_{5}$ and associated with lipid metabolism. ${ }^{13}$ PKAN is also known as neurodegeneration with brain iron accumulation type 1 (NBIA1). In almost $90 \%$ of cases, this condition starts before the age of 6, with rapid progression towards negative outcome within 10 years. PKAN presents with generalised dystonia with buccofacial and lingual involvement, often in the form of severe jaw opening dystonia, although not specifically related to feeding as in chorea-acanthocytosis (online supplementary video 2). ${ }^{14}$ Parkinsonism may be a presenting symptom, and choreoathetosis, tics and pyramidal signs may also occur. About a third of these patients develop retinitis pigmentosa. Sometimes, PKAN has later onset and less severe presentation, with rigidity, focal arm dystonia or cognitivebehavioural onset. ${ }^{14} \mathrm{~A}$ similar orofacial action-specific dystonia, associated with speech and dysarthrophonia, is seen in other forms of NBIA, including Kufor-Rakeb syndrome (autosomal recessive illness caused by ATP13A2 mutations), ${ }^{15}$ aceruloplasminemia and neuroferritinopathy, although the last typically presents in adulthood. ${ }^{5}$ Wilson's disease (WD) may also present with severe orofacial dystonia associated with Parkinsonism and in the setting of a generalised dystonia, thus sometimes posing a diagnostic challenge with HD. Finally, Lesch-Nyhan syndrome can also manifest in the first decade with severe action dystonia and chorea, often involving the bucco-lingual region. ${ }^{16}$

\section{ATAXIA}

Under rare circumstances, HD patients may exhibit a mild degree of cerebellar atrophy throughout disease progression. Marked cerebellar involvement has been reported only very rarely in juvenile HD. When present, cerebellar signs are generally very mild, and the occurrence of clinically more severe cerebellar dysfunction suggests alternative diagnoses.

Probably the most common HD-like syndrome in Caucasian populations is spinocerebellar ataxia 17 (SCA17, also known as HDL4). This accounts for $0.5 \%-1.8 \%$ of all HD-like syndromes, and its age of onset is between 19 and 48 years, rarely starting during childhood. ${ }^{2}$ Like HD, SCA17 is an autosomal dominant trinucleotide-repeat disorder, in which the mutated TBP gene encodes for the TATA-box binding protein, an important general transcription initiation factor. Intergenerational instability, especially during paternal transmission, and anticipation have been recognised. ${ }^{17}$ Cerebellar ataxia is the most common feature, but SCA17 may be very heterogeneous. Dystonia and chorea are the two most frequent movement disorders. When Parkinsonism is present, SCA17 may resemble multiple system atrophy, although without clear autonomic 
dysfunction, and the dopamine transporter single-photon emission computer tomography (DatScan, GE Healthcare) may be abnormal. Pyramidal signs occur in more than a third of cases. Overall, in most families, a true HD-like presentation is observed only in one or few affected members, although intrafamilial phenotypic homogeneity has been described. ${ }^{17}$

Another important HD-like syndrome with cerebellar involvement is DRPLA. This is an autosomal dominant disease caused by CAG-repeat expansion in the ATN1 gene coding for atrophin-1. ${ }^{18}$ Like HD and SCA17, DRPLA shows the phenomenon of anticipation, and its typical age at onset is the third decade. DRPLA presents with three main features, chorea, myoclonus and ataxia. The differential diagnosis with HD and neuroacanthocytosis syndromes can be very challenging, especially when chorea is a predominant clinical feature (online supplementary video 3). When myoclonus is the most disabling symptom, generally this is associated with an earlier onset ( $<20$ years), and with the coexistence of severe progressive myoclonic epilepsy and cognitive decline. When onset occurs after the age of 40 , cerebellar ataxia is usually the predominant characteristic. $^{18}$

Other ataxic syndromes may present rarely with choreatic movements. Unlike in SCA17, chorea presenting in young adults is very uncommon in other autosomal dominant SCAs. Choreatic movements of the limbs are exceptional in SCA1 and SCA2, and slower than in HD. ${ }^{19}{ }^{20}$ Patients with SCA7, another trinucleotide disorder involving the ATXN7 gene coding for ataxin 7 , may rarely manifest small choreatic movements in the distal limbs or in the orofacial district. Rare chorea of the hands has been described in SCA14 patients. ${ }^{21}$ Finally, a recent screening of Greek patients with HD phenocopies identified a CTA*CAG-repeat expansion at the SCA8 locus, involving the complementary base-pairs of the ATXN8OS and ATXN8 genes, the clinical significance of which is however still uncertain. ${ }^{22}$

Autosomal recessive ataxias may present with chorea, but generally onset is in the first two decades. Friedreich ataxia (FA) is by far the most common of these forms, occurring in about 1 in 50000 individuals. FA is characterised by progressive trunk and limb ataxia, absent lower limb reflexes, Babinski sign, dysarthria, reduced vibratory sense and proprioception, visual problems, scoliosis, pes cavus, and cardiomyopathy. Generalised chorea, involving also the tongue and affecting gait and manual dexterity, is a very rare manifestation, with onset in the second decade. $^{23}{ }^{24}$ Apart from the earlier onset, the patients described in these reports differed from typical HD patients in the association with a marked scoliotic abnormality of the spine or with hypertrophic cardiomyopathy, but cerebellar signs were minimal or appeared much later in the course of illness.

Chorea, dystonia and hypomimia occur in 90\% of patients with ataxia-teleangiectasia (AT), starting in the first decade and showing a rapid course. AT is probably the most common form of progressive cerebellar ataxia of early childhood. ${ }^{25}$ This is an autosomal recessive disorder manifesting also with teleangiectasias, immune defects and a predisposition to malignancy. In some patients, the dystonic posturing of the hands in combination with interosseus muscular atrophy may cause characteristic flexion-extension contractures of the fingers. Finally, an earlier onset of dystonia and chorea has been reported in $<15 \%$ of patients with AOA type 1 and type $2 .^{26} 27$

\section{GAIT DISORDER}

Gait examination can be very helpful to identify typical features suggestive of HD-like syndromes. Indeed, most of these syndromes manifest with different motor abnormalities, which may be present in different combination and lead to peculiar gait. The association of chorea and predominantly ataxic gait, with widened base and difficulties performing the tandem gait, is an aspecific feature of both autosomal dominant (SCAs, DRPLA) and autosomal recessive ataxias (FA, AT, AOAs). In choreaacanthocytosis and McLeod syndrome, the co-occurrence of limb chorea, dystonia and peripheral neuropathy contributes to the peculiar gait and postural changes of these disorders. ${ }^{10} 11$ These are characterised by unsteadiness of stance and gait, leading to frequent falls, and caused by impaired postural reflexes, sudden knee buckling, and equinovarus foot deformities secondary to dystonia and atrophy of peroneal muscles. The occurrence of flinging movements of arms and legs, pelvic thrusts and violent trunk spasms further compromise the stability of gait in these patients.

The coexistence of dystonia, rigidity, bradykinesia and spasticity typifies the gait pattern in juvenile HD-like syndromes, for example, PKAN, WD, PLA2G6-associated neurodegeneration (PLAN), Kufor-Rakeb syndrome or the Parkinsonian form of HDL2. ${ }^{3} 1315$ Particularly, gait disturbance is a common presenting symptom in PKAN, originating from the combination of lower limb rigidity, dystonic foot posturing in plantar flexion and inversion, and spasticity, as well as restricted visual field in those children with retinopathy. Marked freezing of gait has also been described in most syndromes mimicking juvenile HD.

\section{EYE MOVEMENT ABNORMALITIES}

A detailed examination of ocular movements can be very informative. HD patients manifest early abnormalities in initiating saccades, which progressively become slow and hypometric, with superimposed dysfunctional gaze fixation. In several progressive $\mathrm{HD}$-like syndromes, primarily chorea-acanthocytosis, ${ }^{11}$ $\mathrm{PKAN}^{14}$ and $\mathrm{WD}^{28}$ we find similar changes. A supranuclear gaze palsy with marked impairment of upgaze saccades, associated with oculogyric dystonic spasms and L-dopa-responsive akinetic-rigid Parkinsonism, is typical of patients with KuforRakeb syndrome. ${ }^{29}$ In chorea-acanthocytosis, fractionated saccades and square-wave jerks are also found. ${ }^{30}$

HD can present also with a mild form of oculomotor apraxia, which is, however, much more severe in AOA types 1 and 2 and AT. ${ }^{25-27}$ When asked to look to one side, subjects with oculomotor apraxia turn their heads first, with eye contraversion, after which their eyes follow to the same side in several slow saccades with head thrusts to the point that, when the head is immobilised, eye movements are virtually impossible.

Finally, it is very important to point out that an early manifestation of 'cerebellar' eye movement abnormalities, for example, dysmetric saccades, square-wave jerks, ocular flutter, saccadic pursuit and gaze-evoked nystagmus, is typical of the most common spinocerebellar degenerative illnesses, and hence very useful to differentiate HD particularly from SCA-17 and FA.

\section{SEIZURES}

In $\mathrm{HD}$, seizures are present only in the juvenile variant, occurring in $30 \%-50 \%$ of patients with onset before age 10 . At difference, in a number of progressive HD-like syndromes seizures are relatively common, presenting in $22 \%$ of SCA-17 cases, $60 \%$ of cases with chorea-acanthocytosis, $40 \%$ of cases with McLeod syndrome ${ }^{31}$ and the majority of cases with Huntington's disease-like 1 (HDL1) syndrome, a prion disease resembling adult-onset HD (detailed below). Seizures are the initial manifestation in almost half of chorea-acanthocytosis cases. Temporal lobe seizures, even simulating a familial 
temporal lobe epilepsy, are seen in chorea-acanthocytosis, whereas they are mostly generalised in McLeod syndrome. ${ }^{31}$ Seizures are also a cardinal feature of DRPLA, present in virtually all cases with onset before the age of 20, less frequently when onset is between ages 20 and 40, and rarely with onset above the age of $40 .{ }^{18}$ Seizures in DRPLA can be tonic, clonic, tonic-clonic, absences, atonic, and in the form of myoclonic epilepsy, with or without a progressive myoclonic epilepsy phenotype. Epilepsy is rare in all the other HD-like syndromes.

\section{SPEED OF PROGRESSION}

The rate of progression of HD can vary considerably across subjects, being, at least in part, related to age of onset and length of trinucleotide-repeat expansion. HD-like syndromes with adult onset may vary in their speed of progression. Importantly, some conditions may progress more rapidly. Among the latter, HDL1, a very rare autosomal dominant prion disease with onset in early adulthood (third-fourth decades), should be kept in account. HDL1 is caused by a 192-nucleotide insertion in a region of the prion protein (PRNP) gene coding for an octapeptide-repeat. HDL1 is recognised as the fourth genetic prion disease phenotype, although not associated typically with spongiform degeneration pathology, but with atrophy and prion deposition within basal ganglia, frontal and temporal lobes, and cerebellar cortex. ${ }^{32}$ Although this condition progresses more slowly than other prion diseases, case series reported that most subjects survive between 1 and 10 years from onset. Patients develop prominent personality changes, cognitive decline, chorea, rigidity, limb and truncal ataxia, dysarthria and seizures. It is useful to remind that chorea may be a feature also of the most common prion disease, that is, Creutzfeldt-Jakob disease. ${ }^{33}$ Other forms characterised by higher speed of progression are HDL2, relentlessly leading to death within 10-20 years, ${ }^{3}$ and DRPLA, generally leading to death within $10-15$ years. ${ }^{18}$

Like juvenile HD, HD-like syndromes with earlier onset progress rapidly. An outstanding exception is benign hereditary chorea (BHC), which, unlike other HD-like syndromes with onset in the first 2 decades, is characterised by a non-progressive or very slowly progressive course dominated by chorea. BHC is now considered a genetically heterogeneous spectrum, first described as an early-onset form of chorea not associated with intellectual deterioration. It is suspected in cases of focal or generalised chorea with autosomal dominant inheritance, and is often described as socially embarrassing (online supplementary video 4). Disease onset occurs in early infancy. A relevant proportion of these cases bear mutations in the NKX2-1 gene encoding for thyroid transcription factor-1 (TITF-1), which is a very important transcription factor involved in the organogenesis of the brain, thyroid and lungs. ${ }^{34}$ An allelic disorder to $\mathrm{BHC}$ presents with a more severe phenotype characterised by choreoathetosis, congenital hypothyroidism and neonatal respiratory distress, also referred to as 'brain-thyroid-lung syndrome', and therefore very different from HD. These cases have also been associated with loss of TITF-1-mediated striatal interneurons. ${ }^{35}$ Given its more benign outcome, it is very important to distinguish $\mathrm{BHC}$ from $\mathrm{HD}$, although not always easy, due to its variable expression. Less frequently than chorea, these patients can manifest intention tremor, dysarthria, gait impairment and exceptionally even psychosis, psychomotor developmental problems and short stature. ${ }^{36}$ Albeit usually non-progressive, the phenotype of $\mathrm{BHC}$ might change with time, shifting from chorea to myoclonus and dystonia. ${ }^{37}$ Reduced striatal volumes on volumetric studies have also been reported. ${ }^{38}$ However, genetic heterogeneity has been observed, with two Japanese families expressing a similar phenotype but with onset between ages 40 and 66, and for this reason resembling adult-onset HD; these subjects, however, lacked cognitive impairment and exhibited an association with a different locus on chromosome $8 \mathrm{q}$. This picture has been named BHC 2, and a postmortem report in one of these patients surprisingly showed pathological changes remarkably similar to those typical of progressive supranuclear palsy (neurofibrillary tangles and tufted astrocytes immunoreactive for four-repeat isoforms of $\tau$ ). ${ }^{39}$

\section{COGNITIVE AND BEHAVIOURAL FEATURES}

Abnormalities of cognitive functioning and behaviour are cardinal features of HD. Classical, adult-onset HD is characterised by a global, progressive cognitive decline. In early stages, cognitive dysfunction may be subtle and detectable mainly through formal neuropsychometry. It may present as loss of mental flexibility, executive dysfunction, memory difficulties (especially of retrieval), and attention or concentration defects. Throughout the course of illness, patients develop forgetfulness, bradyphrenia (ie, slowness of thought processes), impaired visuospatial abilities and ability to manipulate knowledge, and language disturbances leading to reduced syntactic complexity, cortical speech problems, reduced word finding and paraphasic errors. Psychopathology includes early personality changes, depression (which may lead to increased suicidal ideation and suicide risk), hostility, obsessive-compulsive symptoms, anxiety, heightened interpersonal sensitivity and phobias. As disease progresses, patients may exhibit affective or schizophrenic psychoses, particularly with paranoia delusions, as well as explosive and aggressive outbursts, apathy, alcohol abuse, sexual dysfunction and increased appetite.

A formal neuropsychometric and psychiatric assessment may assist in the differential diagnosis in a minority of cases. Some of the most common adult-onset progressive HD-like syndromes are indistinguishable from $\mathrm{HD}$ in terms of their cognitivebehavioural profile. SCA17 leads to a fronto-subcortical dementia in $75 \%-90 \%$ of cases, and to psychiatric symptoms very similar to those in HD in $25 \%-30 \% .{ }^{17}$ The choreatic form of HDL2 presents with a cognitive-behavioural syndrome of milder severity and slower progression, compared with HD and to its Parkinsonian form, in which more severe frontosubcortical dementia, depression, apathy and irritability are observed. ${ }^{3}$ HDL1, on the other hand, is characterised by a more rapid progression of a similar cognitive dysfunction. ${ }^{32}$

A fronto-subcortical dementia is a cardinal feature of DRPLA, present in $90 \%$ of patients, irrespective of their age at onset. Although a psychiatric onset is rare, up to $80 \%$ of DRPLA patients present with behavioural problems. Also, when the onset of DRPLA occurs before the age of 20, we can detect psychotic symptoms, hyperactivity/impulsivity, restlessness, aggressive behaviours, autism-like features, and developmental delay or regression. ${ }^{18}$

The cognitive-behavioural profile of chorea-acanthocytosis is very similar for course and phenomenology to that of classical adult-onset HD, being characterised by a frontal lobe syndrome, apathy, depression, bradyphrenia and obsessive-compulsive symptoms, which are often subtle at onset. Memory impairment, dysexecutive problems and a schizophreniform psychosis may also occur at onset. Some additional features are, however, typical of chorea-acanthocytosis, especially particular compulsive behaviours like trichotillomania, or self-mutilating actions such as chronic head excoriations. Similarly severe, although at an earlier age of onset, the persistent self-injurious behaviours 
of Lesch-Nyhan syndrome consist of biting of fingers, hands, lips and cheeks, and head- or limb-banging against hard objects. Fronto-subcortical cognitive features are milder in McLeod syndrome, whereas psychiatric problems are very common, consisting in personality disorder, anxiety, depression, obsessivecompulsive disorder, and bipolar and schizoaffective disorder. These psychiatric features may precede the movement disorder by many years, whereas cognitive decline appears later in the course of illness.

Juvenile HD differs from adult-onset HD in that it may exhibit a more rapid and severe cognitive deterioration, associated with speech and language delay. The intellectual impairment observed in early onset dystonia/Parkinsonism syndromes, such as PKAN, may be indistinguishable from that of juvenile HD. Atypical forms of PKAN, which begin after the first decade, may be recognised, however, by some cognitive features. Speech problems including palilalia, tachylalia/tachylogia and dysarthria are frequent onset symptoms, which may be associated with personality changes with impulsivity, violent outbursts, depression and emotional lability. Similar features can be observed in all the main metal accumulation disorders resembling juvenile HD, including PLAN, Kufor-Rakeb syndrome and WD. In all these disorders, cognitive-behavioural abnormalities become less severe as age of onset increases.

\section{ANCILLARY INVESTIGATIONS}

Basic ancillary investigations should be performed before any genetic test in the diagnostic work-up of HD-like syndromes. A number of routine blood tests are necessary to differentiate HD from non-progressive causes of chorea. Most of these causes, however, present with an acute or subacute onset, and therefore display a very different course from heredodegenerative HD-like syndromes. A detailed overview of these non-genetic choreas is beyond the scope of this work and has been the object of other review articles. ${ }^{40}$

Some laboratory tests are, however, useful also when facing a clearly progressive hereditary HD-like syndrome. An increased serum activity of creatine kinase and liver enzymes is frequently observed in chorea-acanthocytosis and McLeod syndrome. Low serum ferritin levels can be found in a proportion of patients with neuroferritinopathy, whereas abnormal copper studiesparticularly increased $24 \mathrm{~h}$ urinary copper excretion-support a diagnosis of WD, or aceruloplasminemia if ceruloplasmin is undetectable in the serum. Abnormal lipoprotein electrophoresis can be found in some patients with PKAN. Among autosomal recessive ataxias, hypoalbuminemia is found in AOA type 1, hypercholesterolaemia in both AOA types 1 and 2, whereas increased serum levels of $\alpha$-1-fetoprotein are found in AOA type 2 and AT.

Neuroacanthocytosis syndromes (chorea-acanthocytosis, McLeod syndromes, HDL2, PKAN) are obviously characterised by the presence of increased percentages of acanthocytes in the peripheral blood. However, the determination of an increased acanthocyte percentage in peripheral blood may not be sensitive enough with routine traditional methods, and may require phase contrast microscopy. An additional test that can be used to identify patients with chorea-acanthocytosis is the demonstration of absent chorein expression in erythrocytes using western blotting, but this test is not commercialised and is performed only by very few laboratories in Europe and North America. ${ }^{11}$

Conventional brain MRI may be useful under certain circumstances. The typical adult-onset form of HD is characterised by early striatal atrophy, particularly of the caudate, whereas a certain degree of cortical and cerebellar atrophy may be seen later on in the course of illness. Several adult-onset progressive HD-like syndromes are indistinguishable from HD based on MRI findings. Examples are HDL2, chorea-acanthocytosis and McLeod syndrome. In the last two, the atrophy of the caudate is accompanied by dilatation of the anterior horns of the lateral ventricles, visible particularly on coronal sections, as often seen also in HD. ${ }^{11}$ In addition, $\mathrm{T}_{2}$-weighted high signal lesions in the striatum, mild generalised cortical atrophy (less in McLeod syndrome), and hippocampal sclerosis and atrophy (especially in chorea-acanthocytosis) can be detected. ${ }^{31}$

The presence of cerebellar atrophy at various degrees is very important to help differentiating HD from all forms of SCAs, in particular SCA-17. MRI can be very useful to differentiate DRPLA from HD, in that DRPLA patients exhibit cerebellar and brainstem atrophy, particularly involving the pontine tegmentum, which correlates with age and number of CAG-repeats; ${ }^{18}$ also, diffuse $\mathrm{T}_{2}$-weighted high signal lesions in the deep subcortical white matter can be observed. ${ }^{41}$ Autosomal recessive ataxias like FA, AOA types 1 and 2, and AT may not exhibit cerebellar atrophy early in the course of illness. However, FA may progress with atrophy of the cervical cord, cerebellum and superior cerebellar peduncles, ${ }^{42}$ increased diffusion in white matter tracts on diffusion-weighted imaging, ${ }^{43}$ and reduced $\mathrm{N}$-acetyl-aspartate peak height on magnetic resonance spectroscopy. ${ }^{44}$ BHC, conversely, does not show pathological changes on conventional imaging.

MRI can be of great use in guiding the differential diagnosis between juvenile HD and metal accumulation disorders. PKAN is associated with the pathognomonic 'eye-of-the-tiger' sign, characterised by a central region of hyperintensity surrounded by a rim of hypointensity on coronal or axial $\mathrm{T}_{2}{ }^{*}$-weighted images in the globus pallidus. ${ }^{13} 45$ This abnormality is highly correlated with the presence of PANK2 gene mutations, even in the presymptomatic phase. Other brain metal accumulation disorders, such as PLAN, Kufor-Rakeb syndrome, aceruloplasminemia (iron) and WD (copper) show mainly hypointense pallidal signal without central hyperintensity and, less commonly, iron deposition in the substantia nigra, especially in PLAN and Kufor-Rakeb syndrome. In NBIAs, typically, globus pallidus and substantia nigra appear involved on $\mathrm{T}_{2}{ }^{*}$ and fast spin echo MRI scans, with also dentate involvement seen on $\mathrm{T}_{2}{ }^{*}$-weighted images. ${ }^{45}$ Interestingly, nigral pathology in PLAN and Kufor-Rakeb syndrome seems related to the presence of a PD-like Parkinsonism which responds to L-dopa, and may even be complicated with L-dopa-induced dyskinesias. ${ }^{15} \mathrm{MRI}$, however, may also be completely normal in these disorders.

Finally, other ancillary investigations provide limited additional aid to the diagnostic work-up. The confirmation of a concurrent peripheral neuropathy or myopathy in chorea-acanthocytosis or McLeod syndrome may require a routine electromyographic or electroneurographic study. Evoked potentials may be of some use to demonstrate degeneration of posterior columns in spinocerebellar degenerative ataxias, mainly SCA17 and FA. Muscle imaging, particularly CT, may unveil fatty changes, associated with neurogenic atrophy on biopsy, in patients with chorea-acanthocytosis and McLeod syndrome.

\section{CONCLUSIONS}

The number of progressive heredodegenerative illnesses that need to be considered in patients presenting with HD-like syndrome who tested negative for pathological IT15 gene trinucleotide-repeat expansions has remarkably increased over the next decade. The identification of disease-causing variants in 


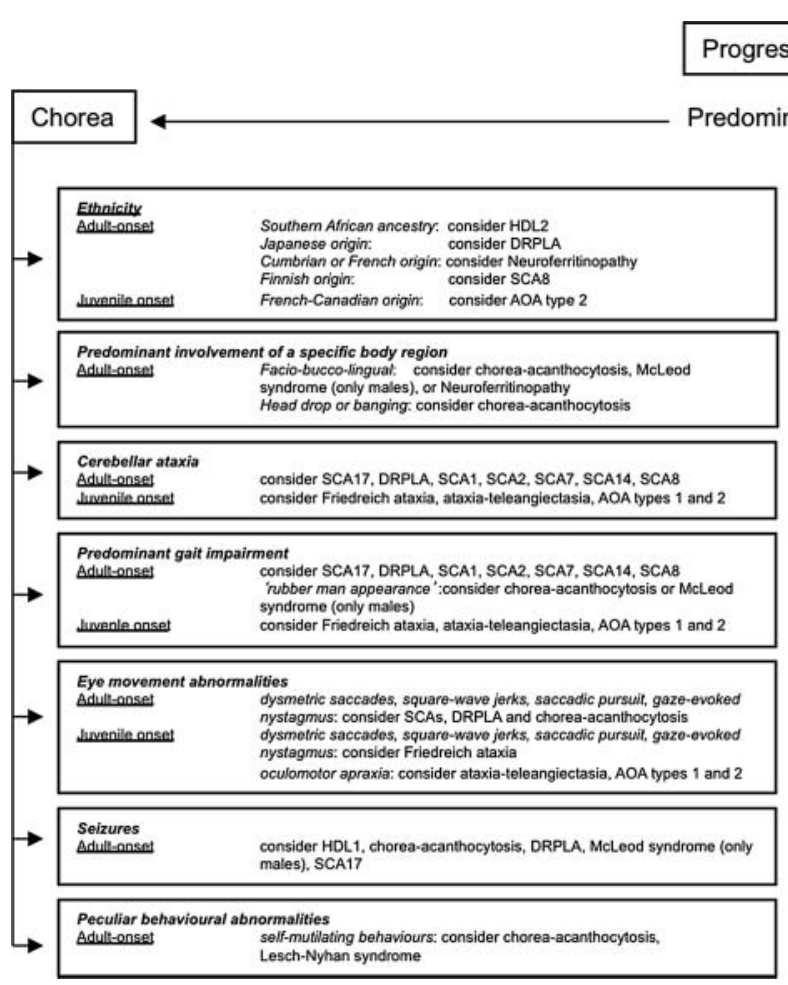

Figure 1 Flowchart summarising 'red flags' for the diagnosis of HD-like (HDL) syndromes that should be considered during bedside examination or in an outpatient setting, presented according to the type of predominant movement disorder. AOA: ataxia with oculomotor apraxia; DRPLA, dentato-rubro-pallido-luysian atrophy; HD, Huntington's disease; PKAN, pantothenate kinase-associated neurodegeneration; PLAN, PLA2G6-associated neurodegeneration; SCA, spinocerebellar ataxia; WD, Wilson's disease.

monogenic disorders is becoming increasingly powerful. Methodologies such as exome sequencing, currently applied on a limited basis, can provide specific genetic diagnoses also in some HD-like syndromes, such as neuroacanthocytosis syndromes, ${ }^{46}$ improving the potential of genotype-phenotype studies. Genetic studies of these diseases remain in general, however, very costly and not widely available in routine diagnostic laboratories. There is a substantial overlap in the clinical phenotype of HD and HD-like syndromes, making the differential diagnosis between these different conditions difficult in several cases. However, a few clinical features, summarised in the flowchart in figure 1 , can help clinicians to rationalise the differential diagnosis, and avoid unnecessary expensive investigations. These red flags represent at present a simple guide that should be constantly updated as the phenotypic characterisation and the identification of more reliable diagnostic markers for HD-like syndromes progress over the following years. The list of novel experimental treatment approaches for HD-like syndromes is also growing. ${ }^{47-49}$ Further understanding of the phenotype-genotype correlation of this heterogeneous group of disorders will help patient selection and outcome prediction with respect to new therapeutic strategies.

Contributors DM: conception and execution of the research project; writing of the first draft. MS and KB: execution of the research project; review and critique of the manuscript. DM: conception and design; acquisition of data; interpretation of data; drafting the article. MS: acquisition of data; critical revision for important intellectual content. KB: conception and design; critical revision for important intellectual content.

\section{Competing interests None.}

Patient consent Obtained.

Provenance and peer review Commissioned; externally peer reviewed.

Open Access This is an Open Access article distributed in accordance with the Creative Commons Attribution Non Commercial (CC BY-NC 3.0) license, which permits others to distribute, remix, adapt, build upon this work non-commercially, and license their derivative works on different terms, provided the original work is properly cited and the use is non-commercial. See: http://creativecommons.org/licenses/by-nc/ 3.01

\section{REFERENCES}

1 Novak MJ, Tabrizi SJ. Huntington's disease: clinical presentation and treatment. Int Rev Neurobiol 2011;98:297-323.

2 Wild EJ, Mudanohwo EE, Sweeney MG, et al. Huntington's disease phenocopies are clinically and genetically heterogeneous. Mov Disord 2008;23:716-20.

3 Margolis RL, Rudnicki DD, Holmes SE. Huntington's disease like-2: review and update. Acta Neurol Taiwan 2005;14:1-8.

4 Martins S, Madama T, Guimaraes L, et al. Portuguese families with dentatorubropallidoluysian atrophy (DRPLA) share a common haplotype of Asian origin. Eur J Hum Genet 2003;11:808-11.

5 McNeill A, Chinnery PF. Neuroferritinopathy: update on clinical features and pathogenesis. Curr Drug Targets 2012;13:1200-3.

6 Ondo WG, Adam OR, Jankovic J, et al. Dramatic response of facial stereotype/tic to tetrabenazine in the first reported cases of neuroferritinopathy in the United States. Mov Disord 2010;25:2470-2.

7 Juvonen V, Vietala M, Kairisto V, et al. The occurrence of dominant spinocerebellar ataxias among 251 Finnish ataxia patients and the role of predisposing large normal alleles in a genetically isolated population. Acta Neurol Scand 2005; 111:154-62.

8 Duquette A, Roddier K, McNabb-Baltar J, et al. Mutations in senataxin responsible for Quebec cluster of ataxia with neuropathy. Ann Neurol 2005;57:408-14.

9 Bader B, Walzer RH, Vogel M, et al. Tongue protrusion and feeding dystonia: a hallmark of chorea-acanthocytosis. Mov Disord 2010;25:127-9.

10 Schneider SA, Lang AE, Moro E, et al. Characteristic head drops and axial extension in advanced chorea-acanthocytosis. Mov Disord 2010;25:1487-91.

11 Jung HH, Danek A, Walzer RH. Neuroacanthocytosis syndromes. Orphanet J Rare Dis 2011;6:68.

12 Gantenbein AR, Damon-Perriere N, Bohlender JE, et al. Feeding dystonia in McLeod syndrome. Mov Disord 2011;26:2123-6.

13 Kurian MA, McNeill A, Lin JP, et al. Childhood disorders of neurodegeneration with brain iron accumulation (NBIA). Dev Med Child Neurol 2011;53: 394-404.

14 Hayflick SJ, Westaway SK, Levinson B, et al. Genetic, clinical, and radiographic delineation of Hallervorden-Spatz syndrome. N Engl J Med 2003;348: 33-40. 
15 Paisan-Ruiz C, Guevara R, Federoff M, et al. Early-onset L-dopa-responsive parkinsonism with pyramidal signs due to ATP13A2, PLA2G6, FBX07 and spatacsin mutations. Mov Disord 2010;25:1791-800.

16 Dabrowski E, Smathers SA, Raistrom CS, et al. Botulinum toxin as a novel treatment for self-mutilation in Lesch-Nyhan syndrome. Dev Med Child Neurol 2005;47:636-9.

17 Stevanin G, Brice A. Spinocerebellar ataxia 17 (SCA17) and Huntington's disease-like 4 (HDL4). Cerebellum 2008;7:170-8

18 Tsuji S. Dentatorubral-pallidoluysian atrophy. Handb Clin Neurol 2012;103:587-94.

19 Namekawa M, Takiyama Y, Ando Y, et al. Choreiform movements in spinocerebellar ataxia type 1. J Neurol Sci 2001;187:103-6.

20 Geschwind $\mathrm{DH}$, Perlman S, Figueroa $\mathrm{CP}$, et al. The prevalence and wide clinica spectrum of the spinocerebellar ataxia type 2 trinucleotide repeat in patients with autosomal dominant cerebellar ataxia. Am J Hum Genet 1997:60:842-50.

21 Stevanin G, Hahn V, Lohmann E, et al. Mutation in the catalytic domain of protein kinase $C$ gamma and extension of the phenotype associated with spinocerebellar ataxia type 14. Arch Neurol 2004;61:1242-8.

22 Koutsis G, Karadima G, Pandraud A, et al. Genetic screening of Greek patients with Huntington's disease phenocopies identifies an SCA8 expansion. J Neurol. Published Online First: 2 February 2012.

23 Zhu D, Burke C, Leslie A, et al. Friedreich's ataxia with chorea and myoclonus caused by a compound heterozygosity for a novel deletion and the trinucleotide GAA expansion. Mov Disord 2002;17:585-9.

24 Spacey SD, Szczygielski Bl, Young SP, et al. Malaysian siblings with Friedreich ataxia and chorea: a novel deletion in the frataxin gene. Can J Neurol Sci 2004;31:383-6.

25 Chun HH, Gatti RA. Ataxia-teleangiectasia, en evolvine phenotype. DNA Repair (Amst.) 2004:3:1187-96.

26 Le Ber I, Moreira MC, Rivaud-Pechoux S, et al. Cerebellar ataxia with oculomotor apraxia type 1: clinical and genetic studies. Brain 2003;126:2761-72.

27 Anheim M, Monga B, Fleury M, et al. Ataxia with oculomotor apraxia type 2: clinical, biological and genotype/phenotype correlation study of a cohort of 90 patients. Brain 2009:132:2688-98.

28 Lorincz MT. Neurologic Wilson's disease. Ann N Y Acad Sci 2010;1184:173-87.

29 Schneider SA, Paisan-Ruiz C, Quinn NP, et al. ATP13A2 mutations (PARK9) cause neurodegeneration with brain iron accumulation. Mov Disord 2010;25:979-84.

30 Gradstein L, Danek A, Grafman J, et al. Eye movements in chorea-acanthocytosis. Invest Ophthalmol Vis Sci 2005;46:1979-87.

31 Scheid R, Bader B, Ott DV, et al. Development of mesial temporal lobe epilepsy in chorea-acanthocytosis. Neurology 2009;73:1419-22.

32 Laplanche JL, El Hachimi KH, Durieux I, et al. Prominent psychiatric features and early onset in an inherited prion disease with a new insertional mutation in the prion protein gene. Brain 1999;122:2375-86.
33 McKee D, Talbot P. Chorea as a presenting feature of variant Creutzfeldt-Jakob disease. Mov Disord 2003;18:837-8.

34 Breedveld GJ, van Dongen JWF, Danesino C et al. Mutations in TITF-1 are associated with benign hereditary chorea. Hum Mol Genet 2002;11:971-9.

35 Kleiner-Fisman G, Calingasan NY, Putt M, et al. Alterations of striatal neurons in benign hereditary chorea. Mov Disord 2005;20:1353-7.

36 Glik A, Vuillaume I, Devos D, et al. Psychosis, short stature in benign hereditary chorea: a novel thyroid transcription factor-1 mutation. Mov Disord 2008;23:1744-7.

37 Armstrong MJ, Shah BB, Chen $\mathrm{R}$, et al. Expanding the phenomenology of benign hereditary chorea: evolution from chorea to myoclonus and dystonia. Mov Disord 2011;26:2296-7

38 Maccabelli G, Pichiecchio A, Guala A, et al. Advanced magnetic resonance imaging in benign hereditary chorea: study of two familial cases. Mov Disord 2010:25:2670-4.

39 Yoshida Y, Nunomura J, Shimomata T, et al. Benign hereditary chorea 2: pathological findings in an autopsy case. Neuropathology. Published Online First: 12 January 2012. doi:10.1111/j.1440-1789.2011.01288.x

40 Cardoso F, Seppi K, Mair KJ, et al. Seminar on choreas. Lancet Neurol 2006:5:589-602.

41 Koide R, Onondera O, Ikeuchi T, et al. Atrophy of the cerebellum and brainstem in dentatorubral pallidoluysian atrophy. Influence of CAG repeat size on MRI findings. Neurology 1997;49:1605-12.

42 Akhlaghi H, Corben L, Georgiou-Karistianis N, et al. Superior cerebellar peduncle atrophy in Friedreich's ataxia correlates with disease symptoms. Cerebellum 2011;10:81-7.

43 Rizzo G, Tonon C, Valentino ML, et al. Brain diffusion-weighted imaging in Friedreich's ataxia. Mov Disord 2011;26:705-12.

44 Ittis I, Hutter D, Bushara KO, et al. (1)H MR spectroscopy in Friedreich's ataxia and ataxia with oculomotor apraxia type 2. Brain Res 2010;1358 200-10.

45 McNeill A, Birchall D, Hayflick SJ, et al. T2* and FSE MRI distinguishes four subtypes of neurodegeneration with brain iron accumulation. Neurology 2008;70:1614-19.

46 Walzer RH, Schulz VP, Tikhonova IR, et al. Genetic diagnosis of neuroacanthocytosis disorders using exome sequencing. Mov Disord 2012;27:539-43.

47 Evers MM, Pepers BA, van Deutekom JC, et al. Targeting several CAG expansion diseases by a single antisense oligonucleotide. PLoS One 2011;6: e24308.

48 Hartig MB, Prokisch $\mathrm{H}$, Meitinger $\mathrm{T}$, et al. Pantothenate kinase-associated neurodegeneration. Curr Drug Targets 2012;13:1182-9.

49 Edwards TC, Zrinzo L, Limousin $\mathrm{P}$, et al. Deep brain stimulation in the treatment of chorea. Mov Disord 2012;27:357-63. 